\title{
A Pan-STARRS1 Search for Substellar Young Moving Group Members
}

\author{
Kimberly M. Aller ${ }^{1}$, Michael C. Liu ${ }^{2}$ and Eugene A. Magnier ${ }^{3}$ \\ ${ }^{1}$ University of Hawaii, Institute for Astronomy \\ email: kaller@ifa.hawaii.edu \\ ${ }^{2}$ University of Hawaii, Institute for Astronomy \\ email: mliu@ifa.hawaii.edu \\ ${ }^{3}$ University of Hawaii, Institute for Astronomy \\ email: eugene@ifa.hawaii.edu
}

\begin{abstract}
Young moving groups (YMGs) are coeval, comoving groups of stars which have migrated from their birthsites after formation. In the substellar regime, YMG members are key benchmarks to empirically define brown dwarf evolution with age and to study the lowest mass end of the initial mass function. We have combined Pan-STARRS1 (PS1) proper motions with optical+IR photometry from PS1, 2MASS and WISE to perform a large-scale $\left(\approx 30,000 \mathrm{deg}^{2}\right)$ systematic search for substellar members down to $\approx 10 M_{\text {Jup }}$. We have obtained near-IR spectroscopy of a large sample of ultracool candidate YMG members to assess their youth via gravity-sensitive absorption features. We have identified several new intermediate-gravity candidate members of the AB Dor Moving Group, potentially greatly expanding the substellar membership. These new candidate members bridge the gap between the known low-mass stellar and planetary-mass members and yield valuable insight into the spectral characteristics of young brown dwarfs.
\end{abstract}

Keywords. brown dwarfs, low-mass stars

\section{Introduction}

In the past 15 years, large astrometric surveys such as Hipparcos (Perryman et al. 1997) have been used to discover young ( $\lesssim 100 \mathrm{Myr}$ ), coeval stellar associations within $100 \mathrm{pc}$, which have left their molecular cloud after formation and dispersed into the field (e.g. Zuckerman \& Song 2004). These young moving groups (YMGs) provide a valuable evolutionary link between ongoing star formation in molecular clouds ( $\sim 1 \mathrm{Myr})$ and old field stars ( $>1$ Gyr). Because YMGs are relatively nearby $(\lesssim 100 \mathrm{pc})$, they are ideal for characterizing the mass function down to substellar masses. In particular, the substellar YMG members are valuable benchmarks for establishing the relationship between spectral type, age, and luminosity.

Photometry and spectroscopy of young brown dwarfs and directly imaged planets have revealed that they differ from their old field counterparts (e.g. Chauvin et al. 2005; Marois et al. 2008; Bowler et al. 2010, 2013; Patience et al. 2010; Barman et al. 2011). Young brown dwarfs also have redder NIR colors compared to field brown dwarfs of the same spectral type (e.g. Gizis et al. 2012). Recent studies have begun to classify gravitysensitive spectral features by comparing young and old ultracool dwarfs (e.g. Allers et al. 2007; Allers \& Liu 2013). A key step towards further characterizing brown dwarf spectral evolution is to determine the substellar spectral sequence in YMGs spanning the ages of 10-100 Myr. However, until the past few years, very few bona fide brown dwarf YMGs members were known. 


\section{Data and Results}

We have used Pan-STARRS1 (PS1) proper motions and photometry from PS1, 2MASS and WISE to conduct a large-area search for substellar members of YMGs. PS1 is a unique resource for such work because its large area coverage $\left(30,000 \mathrm{deg}^{2}\right)$ encompasses the sparsely distributed YMGs; its far-red sensitivity enables detection of $\sim 10 \times$ more objects than 2MASS; and its multi-epoch high quality astrometry can distinguish candidate YMG members from old field stars. We use PS1, 2MASS and WISE photometry to construct spectral energy distributions and then estimated spectral types and photometric distances using the template-fitting method of Aller et al. (2013). We then used PS1+2MASS astrometry to determine proper motions of our candidate YMG members and followed the methods of Schlieder et al. (2012) to screen for candidates with space motions consistent with the known YMGs.

We have obtained follow-up near-IR spectroscopy of several candidates using SpeX (Rayner et al. 2003), a medium-resolution near-IR spectrograph $(0.8-2.5 \mu \mathrm{m})$ on the 3-meter NASA Infrared Telescope Facility (IRTF). We used the Allers \& Liu (2013) classification method to assess the spectral type and youth of our candidates. On this system, spectral types are determined by combining a visual comparison of our objects to the Kirkpatrick et al. (2010) spectral standards in the $J$ and $K$ bands with the index-based methods from McLean et al. (2003), Slesnick et al. (2004), and Allers et al. (2007). We discovered two early-L dwarfs $\left(\approx 30-40 M_{J u p}\right)$ and two late-M dwarfs $\left(\approx 60-70 M_{J u p}\right)$ as candidate substellar members of the AB Dor Moving Group, with intermediate gravities typical of young ultracool dwarfs. We also discovered two other L dwarf candidate AB Dor Moving Group members with spectra that show hints of youth but may be older field objects. Figure 1 shows the NIR spectra of our candidate AB Dor Moving Group members. In Figure 2, we compare one of our AB Dor Moving Group candidates, which we have classified as intermediate gravity (INT-G) on the Allers \& Liu (2013) system, with an old field gravity (FLD-G) standard object from Kirkpatrick et al. (2010) and a young, very low gravity (VL-G) standard object from Allers \& Liu (2013).

Although the known young and dusty field L dwarfs have redder WISE colors (W1W2) and NIR colors (J-K) compared with old field dwarfs of the same spectral type (e.g. Gizis et al. 2012), not all of our AB Dor candidates appear to follow this trend. One possible reason that our AB Dor Moving Group candidates members are not significantly redder than their field dwarf counterparts is that they are older $(\sim 125 \mathrm{Myr})$ than the brown dwarf members of the TW Hydrae or $\beta$ Pic moving groups ( $\sim 10-20$ Myr), which have gravity classifications of VL-G on the Allers \& Liu (2013) system.

\section{Conclusions}

We have combined PS1, 2MASS, and WISE photometry with PS1+2MASS proper motions to systematically search for substellar YMG members. We have spectroscopically confirmed the youth of four candidate members of the AB Dor Moving Group and have two other candidate members with tentative spectroscopic signatures of youth. Our AB Dor Moving Group candidate members have NIR colors which are not significantly redder than their field counterparts, suggesting that intermediate-aged (INT-G) brown dwarfs may not be as red as young (VL-G) brown dwarfs of the same spectral type. Our candidate young brown dwarf AB Dor Moving Group members are benchmarks which can be used to characterize the brown dwarf spectral evolution and to study the atmospheres of young exoplanet analogs. 


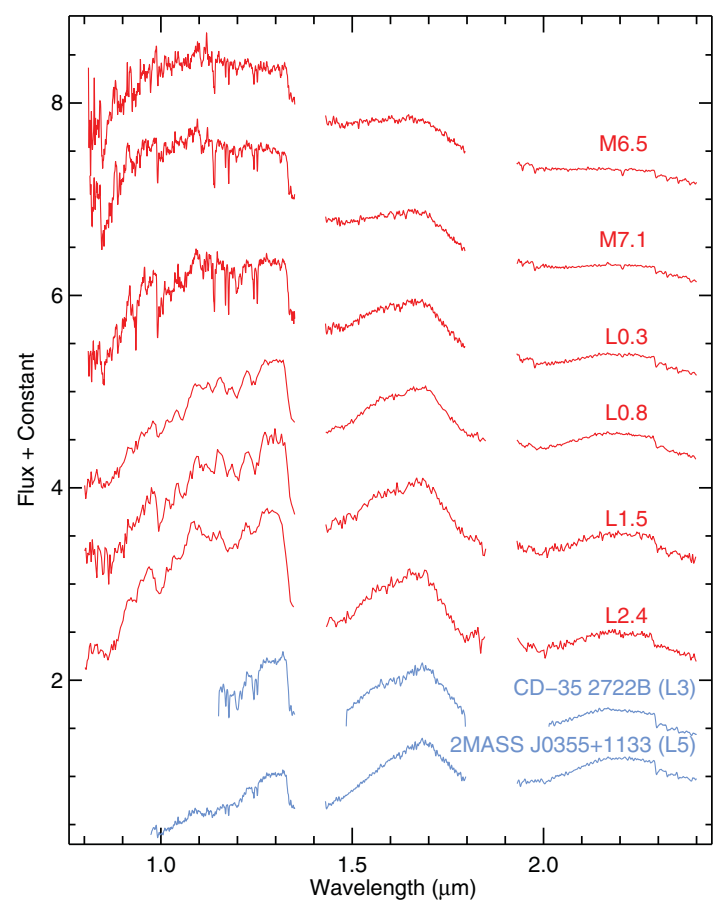

Figure 1. NIR spectra of our AB Dor Moving Group candidate members (red) compared to two known brown dwarf members (blue), CD-35 2722B (Wahhaj et al. 2011) and 2MASS J0355+1133 (Liu et al. 2013a; Faherty et al. 2013). The spectral types of our candidates are determined by combining visual and index-based classifications as in Allers \& Liu (2013).

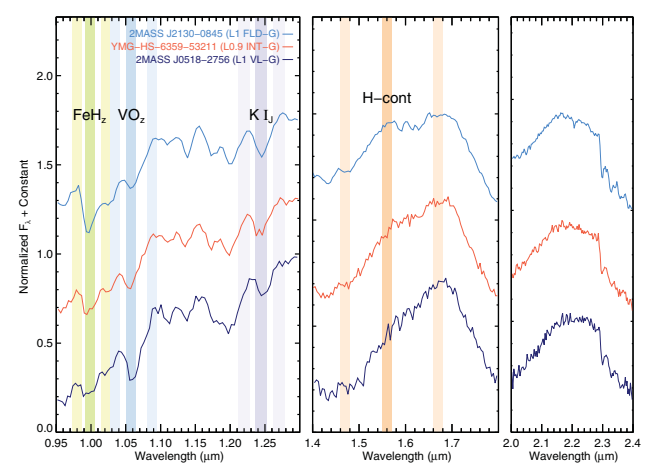

Figure 2. NIR low-resolution $(\mathrm{R} \sim 130)$ spectra from IRTF/SpeX of one of our AB Dor Moving Group candidates (INT-G, red) compared with a field (FLD-G, light blue) and a young (VL-G, dark blue) dwarf with a spectral type within half a spectral type from our candidate. We used a field spectral standard from Kirkpatrick et al. (2010) and a young standard from Allers \& Liu (2013) smoothing all spectra to a common resolution. Wavelength regions used to calculated gravity-sensitive indices from Allers \& Liu (2013) are highlighted for $\mathrm{FeH}_{z}$ (yellow-green), $\mathrm{VO}_{z}$ (blue), $\mathrm{KI}_{J}$ (purple), and $\mathrm{H}$-cont (orange).

\section{References}

Aller, K. M., et al. 2013, ApJ, 773, 63

Allers, K. N. \& Liu, M. C. 2013, ApJ, 772, 79

Allers, K. N., et al. 2007, ApJ, 657, 511

Barman, T. S., Macintosh, B., Konopacky, Q. M., \& Marois, C. 2011, ApJ, 733, 65 
Bowler, B. P., Liu, M. C., Dupuy, T. J., \& Cushing, M. C. 2010, ApJ, 723, 850

Bowler, B. P., Liu, M. C., Shkolnik, E. L., \& Dupuy, T. J. 2013, ApJ, 774, 55

Chauvin, G., Lagrange, A.-M., Dumas, C., Zuckerman, B., Mouillet, D., Song, I., Beuzit, J.-L., \& Lowrance, P. 2005, A\&BA, 438, L25

Faherty, J. K., Rice, E. L., Cruz, K. L., Mamajek, E. E., \& Núñez, A. 2013, AJ, 145, 2

Gizis, J. E., et al. 2012, AJ, 144, 94

Kirkpatrick, J. D., et al. 2010, ApJS, 190, 100

Liu, M. C., Dupuy, T. J., \& Allers, K. N. 2013a, Astronomische Nachrichten, 334, 85

Marois, C., Macintosh, B., Barman, T., Zuckerman, B., Song, I., Patience, J., Lafrenière, D., \& Doyon, R. 2008, Science, 322, 1348

McLean, I. S., McGovern, M. R., Burgasser, A. J., Kirkpatrick, J. D., Prato, L., \& Kim, S. S. 2003, ApJ, 596, 561

Patience, J., King, R. R., de Rosa, R. J., \& Marois, C. 2010, A\&A, 517, A76

Perryman, M. A. C., et al. 1997, A\&JA, 323, L49

Rayner, J. T., Toomey, D. W., Onaka, P. M., Denault, A. J., Stahlberger, W. E., Vacca, W. D., Cushing, M. C., \& Wang, S. 2003, PASP, 115, 362

Schlieder, J. E., Lépine, S., \& Simon, M. 2012, AJ, 143, 80

Slesnick, C. L., Hillenbrand, L. A., \& Carpenter, J. M. 2004, ApJ, 610, 1045

Wahhaj, Z., et al. 2011, ApJ, 729, 139

Zuckerman, B. \& Song, I. 2004, ARA\&A, 42, 685 